\title{
Matter of Similarity and Dissimilarity in Multi- Ethnic Society: A Model of Dyadic Cultural Norms Congruence
}

\author{
Hassan Abu Bakar ${ }^{1, *}$, Bahtiar Mohamad ${ }^{1}$ \\ ${ }^{1}$ Department of Communication, School of Multimedia Technology \& Communication, 06010 UUM \\ Sintok, Kedah, Malaysia
}

\begin{abstract}
Taking this into consideration of diver cultural norms in Malaysian workplace, the propose model explores Malaysia culture and identity with a backdrop of the pushes and pulls of ethnic diversity in a Malaysia. The model seeks to understand relational norm congruence based on multiethnic in Malaysia that will be enable us to identify Malaysia cultural and identity. This is in line with recent call by various interest groups in Malaysia to focus more on model designs that capture contextual and cultural factors that influences Malaysia culture and identity.
\end{abstract}

\section{Introduction}

In Malaysia, ethnic divisions are coterminous with its linguistic, cultural, religious and economic differences. Malaysian society, individuals hold differing cultural orientations, norms and values according to their ethnicity (i.e., ethnic Malays, ethnic Chinese, ethnic Indian). While there is very little cultural homogeneity between the three ethnicities in Malaysia, their divergent value differences converge under the nation of Malaysia. This convergence can be considered as contributing to the unique face of Malaysian identity. Thus, we see ethnic divisions in the nature of work, occupations and social structure in Malaysia. For example, a majority of Bumiputra work in manufacturing and the public sectors, the Chinese dominate management and professional positions along with a small number of Indians, and a majority of Indians work in the plantation sector. Such unique heterogeneity (with homogeneous subdivisions) helps highlight the complexity of cultural norms in Malaysia, However, to date not many studies have address this research lacuna, therefore this study will explore the Malaysia identity and culture from various perspectives.

Taking this into consideration, the propose study will explore Malaysia national culture and identity with a backdrop of the pushes and pulls of ethnic diversity in a Malaysia. This article seeks to understand relational norm congruence based on multiethnic in Malaysia that will be enable us to identify Malaysia cultural and identity. This is in line with recent

* Corresponding author: abhassan@uum.edu.my 
call by various interest groups in Malaysia to focus more on research designs that capture contextual and cultural factors that influences Malaysia culture and identity. In paper we question how cultural relational norms and values in Malaysia may provide conditions for the congruence model of multiethnic society in Malaysia? and we believe this is a fundamental question that deserves further systematic investigation. We feel that this salience question to explore characteristic of the Malaysian national cultural and identity.

\section{Theory and Model Development}

Malaysia is a multiethnic society in which three major ethnic groups (Malays/Bumiputra, Chinese, Indian) interact with a significant degree of harmony in their everyday living. Each of these ethnic groups has also, to a large degree, managed to preserve their divergent ethnic identities via continued practice of customs, behavior, languages spoken, norms, values, and beliefs. Malaysia's society and work force has been found to be reflective of this cultural, behavioral, and value diversity. While there is very little cultural homogeneity between the three ethnicities in Malaysia, their divergent value differences converge under the nation of Malaysia. This convergence can be considered as contributing to the unique face of "Malaysian identity". However, to date not many studies have addressed this research lacuna, therefore this study will explore the Malaysia identity and culture from various perspectives. For this reason, we argue that understanding and developing Malaysia national cultural and identity is a significant and powerful issue, and can be viewed as a salient issue for diverse multiethnic society like Malaysia. In fact, to date little is known about what underlies Malaysia national culture and identity in a truly multiethnic society like Malaysia. Thus, this offers unique opportunity for investigation as aims to explore Malaysia national culture and identity by capturing diversity in a society that is inherently diverse. Our proposition here is in line with a recent call by various interest groups in Malaysia to focus more on research designs that capture contextual and cultural factors that influences Malaysia culture and identity that will lead to national unity. We question how cultural relational norms and values in Malaysia may provide conditions for the congruence model of multiethnic society in Malaysia? And we believe this is a fundamental question that deserves further systematic investigation. We feel that this salience question to explore characteristic of the Malaysian national cultural and identity.

Two conceptual frameworks underlie most ethnicity and cultural identity research [1]. The first of these is similarity-attraction/identification, while the second is relational norm congruence. Similarity-attraction theory was largely developed devoid of cultural considerations and is based on social cognitive processes of "like attraction" [2], social identification [3, 4], and self-categorization [5], The general idea behind similarityattraction is that people like (and are attracted to) others who are similar (vs. dissimilar) to themselves. This "birds of a feather flock together" concept posits that similarity leads to interpersonal attraction and shared group identity, which produces in-group favoritism or positive bias. These biases and favoritisms are then associated with various relational and task-related outcomes. Social scientific research has provided significant support for tenets of similarity attraction theory since the mid-1900s. A. S. Tsui [6] argued that similarity in relationships demographic attributes would be associated with more positive outcomes and affect toward multiethnic relationships, and less role ambiguity and conflict. Their empirical test was partially supported. To more fully understand relational demography and its impact on workplace interactions, later studies went beyond social cognitive explanations and focused on our second underlying theory - relational norm congruence [7, 8]. Relational norm congruence specifies that multiethnic demographic similarity or dissimilarity, when congruent with the cultural norm for that demographic category, is 
associated with more positive relational and task outcomes [9]. It is worth noting that while perceived similarity has been shown to perform better than relational demographic variables in many Western contexts, some research suggests that they may be less useful in non-Western settings where relational norm congruence is a key concern [10]. Research has shown that the effect of relational demography is, to a large extent, a function of the relational norms of a particular culture [11]. To further understand this dyadic (dis)agreement in a particular cultural context, we argue that it is necessary to incorporate relational demography as an important factor in our analysis. This is especially true in cultures where relational norm congruence and societal cultural norms are best characterized by relational demography. Thus, we see that understanding relational norm congruence based on multiethnic in Malaysia will be enabling us to identify Malaysia cultural and identity. This can be done through identifying similarity and dissimilarity characteristics of cultural and identity that are congruence with norms of each ethic in Malaysia.

Hierarchical differentiation is an important part of ongoing interpersonal interactions. Without question, the supervisor plays a large role in shaping a subordinate's "organizational reality," and in many ways the supervisor can be the face of the organization for the subordinate. The supervisor is often the information provider to the subordinate, the connection to the "upper ranks" of the organization, and the provider of the subordinate's job definition and realities. The supervisor-subordinate relationship quality (ranging from low quality to high quality relationship exchange) can affect a supervisor's decisions concerning resource allocation, delegation of responsibilities, and evaluative information about job behavior (i.e., performance evaluations). All of this can have a profound effect on the subordinate because of their lower hierarchical status in the dyad. Taking the above into consideration, we argue that the dynamics (in terms of demographic diversity) that help shape supervisor-subordinate relationship quality exchanges will eventually affect subordinate perceptions of the exchanges, as well as attitude about the job itself [12]. While we recognize that several studies have found simple employee demographic characteristics to correlate with outcomes such as satisfaction, commitment and performance $[13,14]$ these studies do not connect demographic diversity variables in a specific cultural context. Therefore, this study will focus our investigation of demographic attributes that are tied closely to the Malaysian organizational context. They include (dis)similarities in ethnicity, religion, gender, age, and tenure. In the following paragraphs, we develop hypotheses with regard to each attribute, respectively. Ethnicity and Religion Malaysian society is primarily comprised of the following ethnic groups; Malay $50.4 \%$, Chinese $23.7 \%$, indigenous $11 \%$, Indian $7.1 \%$, others $7.8 \%$ [15]. Each of these ethnic groups maintains their own strong ethnic identity and their own cultural customs, practices, language, values and beliefs [16]. Religion is closely tied to ethnicity in Malaysia. For example, the Malays are Muslims, most Indians follow Hinduism, and the majority of Chinese practice a combination of Taoism and Buddhism, mixed with values associated with Confucianism. A small minority of Indian and Chinese follow Christianity and Islam, respectively [17, 18]. Unlike most Western societies where religion is kept out of the workplace, Malaysian work settings readily accommodate those observing various religious rituals and practices [17]. In sum, ethnic difference is a salient relational demographic attribute actively maintained and highly visible in both secular and religious affairs [19]. Perhaps not surprisingly, when team building in Malaysian organizations, strong ethnic identities tend to cultivate in-group favoritism and trust [18]. As such, managers may be more likely to prefer members from the same ethnic group as we would expect from the similarity attraction, social identity and self-categorization arguments discussed earlier. Motivated by self-enhancement and uncertainty-reduction, ethnic similarity reinforces a stable social identity and facilitates in-group social interaction. According to social 
exchange theory [20], reciprocity - as a primary basis of exchange - is culturally conditioned. Leader-member dyads with the same ethnic identity share similar norms of reciprocity and are more likely to achieve mutual agreement with regard to the levels of exchange resources, such as organizational commitment, job satisfaction and performance ratings. In contrast, leader-member dyads comprised of those with differing ethnic identities are more likely to have different cultural expectations, and are also likely to have difficulties in achieving agreement about their relationship quality. As mentioned, religion is closely related to ethnicity in Malaysia. Unlike what one might see in many Western societies, religion in Malaysian workplaces is visibly practiced and highlights differences in cultural values. Similar to ethnicity, then, we would expect that dyads sharing the same religion would find it easier to achieve agreement and reciprocate benefits and obligations. Prior research speaks to this point, as similar dyads in ethnicity were found to reinforce members' perceptions of agreement about organizational values [14] and contribute to performance [21]. Hence, we predict that:

Proposition 1: Within the ethnic similarity dyad will be positively associated with the dyad relationships quality ratings

Proposition 2: Within the ethnic dissimilarity dyad will be negatively associated with the dyad relationships quality ratings

\section{Limitation and Future Directions}

Although the proposed model has advanced our knowledge about relationships in diverse workplace, a few limitations of this study need to be noted. First, when interpreting the proposed model, one must recognize that the model was limited to a single country. While we strongly feel that this multi-ethnic organization is quite typical of large Malaysian businesses, this does remain the model limitation. Second, statements of causality based on the positive or negative relationships must be treated with caution given the co-relational nature of the variables. Future research might examine the relational demography paradigm within a variety of organizations that have different demographic distributions (e.g., expatriates working in Malaysian organizations) to increase the generalizability of the present model. More cultural variables should be considered. Third, our current model limits itself to similarity and dissimilarity and relationships outcome variables. These outcome variables are an important component of organizations, and they are interrelated. Therefore, the correlation between relational demographic and outcome variables must be treated with caution. While the model has uncovered many useful findings, future studies may include other outcome variables such task delegation and absenteeism. Lastly, the present study was limited to five demographic variables. Future research should try to include as many demographic characteristics as possible, including work group context and educational differences. In summary, despite this model's limitations, the model herein unquestionably extends our understanding of relational demography and supervisorsubordinate relationships quality. We have done so by identifying specific demographic characteristics as important components of the social context in which the dyad operates in a workgroup. While the pattern of the model raises some questions about the generalizability of the relational demography paradigm to all demographic characteristics, our model strongly suggests that similarities in the dyad are critical to the dyad relationship and work attitudes. In order to continue providing knowledge useful for organizations and their managers, we urge researchers to continue their efforts to identify specific demographic characteristics within the supervisor-subordinate relationship and work group that may influence work relationships outcomes. 


\section{References}

1. M. M. Steward, Garcia-Prieto, J Organ Behav, 29 (2008)

2. D. Byrne, The attraction paradigm. (Academic Press, New York, 1971)

3. B. E. Ashforth, F. A. Mael. ACAD MANAGE REV, 14, (1989)

4. M. A. Hogg, D. Abrams, D. Social identifications: A social psychology of intergroup relations and group processes. (Routledge, London 1988)

5. M. A. Hogg, D. J. Terry. (2000). ACAD MANAGE REV, 25, (2008)

6. A. S. Tsui, C. A. O'Reilly. ACAD MANAGE J, 32, (1989)

7. R. C. Liden. Asia Pacific Journal of Management, 28, (2011)

8. R. P. Vecchio. D. M. Brazil. Personnel Psychology, 60, (2007).

9. C. Goldberg. C. M. Riordan. L. Zhang. Group \& Organization Management, 33, (2008)

10. A. S. Tsui. H. Wang. K. R. Xin. Management \& Organization Review, 2 (2006)

11. D. C. Lau. L. W. Lam. S. D. Salamon. Journal of Social Psychology, 148 (2008)

12. M. A. Wesolowski. K. W. Journal of Organizational Behavior, 18 (1997)

13. D. R. Avery. P. F. McKay. D. C. Wilson. Journal of Applied Psychology, 92 (2007)

14. D. R. Avery. P. F. McKay. D. C. Wilson. Personnel Psychology, 60 (2007)

15. K. T. Bhal. N. Gulati, N. M. A. Ansari. LEADERSHIP ORG DEV J, 30 (2009)

16. Abdul Rashid, M. Z., Sambasivan, M., \& Abdul Rahman, A. LEADERSHIP ORG DEV J, 25 (2004)

17. A. Abdullah. S. Singh. S. K. Gill. Understanding the Malaysian workforce: Guidelines for managers. (Malaysian Institute of Management, Malaysia, 2001)

18. K. Ahmad, K. International Journal of Commerce \& Management, 11 (2001)

19. C. Selvarajah. D. Meyer. Leadership \& Organization Development Journal, 29 (2008)

20. R. Cropanzano. M. S. Mitchell. J MANAGE, 31 (2005).

21. P. F. McKay. D. R. Avery. M. A. Morris. Personnel Psychology, 61 (2008) 